\title{
Prognostic impact of tertiary lymphoid structures in breast cancer prognosis: a systematic review and meta-analysis
}

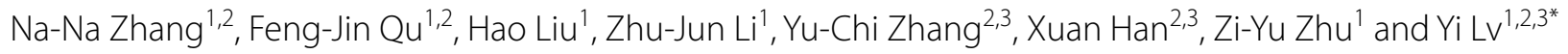

\begin{abstract}
Background: Tertiary lymphoid structures (TLSs), organizationally resemble lymph nodes, are frequently present in breast cancer (BCa). It is usually, but not always, associated with a positive prognosis or immunotherapy response in cancer patients. This meta-analysis was performed to assess the prognostic and clinical impact of TLSs in BCa.

Methods: We conducted a systematic search in PubMed, Embase, Cochrane Library, Web of Science, China National Knowledge Infrastructure, and WanFang Database to obtain eligible research data up to May 30, 2021. This meta-analysis is focusing on the studies evaluated the prognostic value of TLSs and the associated clinicopathologic indicators, related gene expression and survival. STATA software 16.0 software was used to assess the prognostic significance and clinical impact of TLSS.
\end{abstract}

Results: Nine studies involved with 2281 cases were incorporated in this meta-analysis, in which four of them evaluated the prognostic value of TLSs. There are 6 studies assessed the relationship of TLSs and 4 studies investigated the clinicopathologic parameters as well as the key gene expression, respectively. The results showed the presence of TLSs were predicting a better OS ( $\mathrm{HR}=0.61,95 \% \mathrm{Cl}: 0.51-0.73, p<0.001)$ and DFS ( $\mathrm{HR}=0.40,95 \% \mathrm{Cl}: 0.17-0.93$, $p<0.001)$ of BCa patients. It also revealed that the presence of TLSs was significantly correlated with tumor differentiation ( $p<0.001)$, pTNM stage $(p<0.001)$, lymph node metastasis $(p<0.001)$, and TILs density $(p<0.001)$ of BCa, and the expression of Her2 $(p<0.001)$, ER $(p<0.001)$, PR $(p<0.001)$ and Ki67 $(p=0.009)$ of the tumor cell.

Conclusion: Our results indicated that high levels of TLSs could predict a favorable prognosis for BCa. Moreover, the TLSs were significantly correlated with the clinicopathological indicators and the critical gene expression of $\mathrm{BCa}$, indicating its potential clinical impact on BCa patients.

Keywords: Tertiary lymphoid structures, Breast cancer, Prognosis, Immunotherapy, Meta-analysis

\section{Introduction}

Breast cancer $(\mathrm{BCa})$ is the most commonly diagnosed malignancy in females, imposing pressing scientific, clinical, and societal challenges. The incidence of $\mathrm{BCa}$ is

\footnotetext{
*Correspondence: luyi169@126.com

${ }^{1}$ Center for Regenerative and Reconstructive Medicine, Med-X Institute of Western China Science and Technology Innovation Harbour, The First Affiliated Hospital of Xi'an Jiaotong University, Xi'an 710049, Shaanxi, China

Full list of author information is available at the end of the article
}

rising in most countries and is projected to increase further over the next 20 years despite dramatic efforts for its prevention and treatment. The American Cancer Society has estimated that nearly $30 \%$ of new cancer cases and $15 \%$ of cancer deaths among women could be caused by BCa [1-3].

Recently, exciting progress in cancer immunotherapy has ushered in a new era in cancer treatment. Immune checkpoint blockade (ICB) therapy has already improved patient survival in various cancers $[4,5]$. However, not all cancer patients benefit 
from immunotherapy. Studies have reported that only approximately $5 \%$ of patients with metastatic triplenegative $\mathrm{BCa}$ obtain a positive response to $\mathrm{PD}-1 /$ PD-L1 blockade [6, 7], and the response rate seems to increase to $19-23 \%$ upon selection of patients with a PD-L1-positive tumor microenvironment (TME) [8, 9]. Therefore, investigation of the specific factors contributing to the cancer response to immunotherapy and of the corresponding biomarkers predicting the utility and effect of immunotherapy is of great significance for cancer patients [10].

Tertiary lymphoid structures (TLSs) are ectopic lymphoid organs formed in nonlymphoid tissues during chronic inflammation and tumorigenesis. TLSs identified from several solid tumors have been verified to be positively correlated with the response of cancer patients to immunotherapy [11, 12]. In melanoma, the densities of CD20 + B cells and TLSs in ICB responders' tumor tissues were found to be significantly higher than those in nonresponders [13]. Many studies have indicated that immune cells within TLSs can improve tumor antigen presentation, increase cytokine-mediated signaling, and stimulate CD8 $+\mathrm{T}$ cells to attack tumor cells $[10,13]$. TLSs are essential sites for initiating and maintaining local and systemic $\mathrm{T}$ and $\mathrm{B}$ cell responses against tumors. In addition, TLSs are a privileged site for the recruitment of lymphocytes into tumors and the generation of central memory $\mathrm{T}$ and $\mathrm{B}$ cells, which can circulate and slow cancer progression [14]. Thus, TLSs can be considered a novel biomarker to stratify untreated cancer patients' survival risk. TLSs are also expected to be a very promising quantitative biomarker for predicting the efficacy of tumor immunotherapy in the future.

Although many studies have reported the positive effect of TLSs on the prognosis of BCa patients [10, 12, 13], the relationships between TLSs and patients prognosis, the expression of associated genes, and other clinicopathological parameters are inconsistent among studies and are even contradictory among samples from patients of different races and detection methods adopted by the researchers $[15,16]$. To determine the prospective clinical significance of TLSs, it is essential to clarify the role of TLSs and their relationship with other parameters related to patient prognosis. Thus, this literature-based meta-analysis was conducted to identify a much more specific prognostic value of TLSs and a clear relationship between TLSs and prognosis-related parameters. This comprehensive analysis may provide crucial information on the tumor microenvironment's immune status and its dynamic effect on tumor progression, possibly leading to a more powerful, strengthened biosignature for predicting the clinical outcomes and sensitivity to immunotherapy of BCa patients.

\section{Materials and methods}

\section{Search strategy}

Two authors (NNZ and FJQ) were responsible for independently searching the comprehensive databases and evaluating the availability of studies in PubMed, Embase, the Cochrane Library, Web of Science, China National Knowledge Infrastructure (CNKI) and the WanFang database to obtain eligible studies published before May 30, 2021. The search terms included tertiary lymphoid organ (TLO), tertiary lymphoid structures (TLSs), tertiary lymphoid tissue (TLT), ectopic lymphoid-like structures (ELSs), breast cancer/tumor/malignancy, and prognosis, prognostic or survival outcome. The exact search query is provided as follows to allow reproducibility: (TLO OR TLSs OR TLT OR ELSs) AND (breast cancer OR breast tumor OR breast malignancy) AND (prognosis OR prognostic OR survival outcome). There were no other limitations in the process for searching the databases. Moreover, we reviewed the references in relevant articles to find potential studies. Two researchers independently screened titles and abstracts based on the inclusion and exclusion criteria. Finally, we selected studies that needed reference data through full-text reading. Any differing opinions between the two researchers were resolved through discussion with more researchers.

\section{Study selection}

Original studies eligible for inclusion in this meta-analysis satisfied all of the following criteria: (1) studies focusing on patients with breast cancer (BCa); (2) studies investigating the TLSs in situ in tumor tissue by applying H\&E staining and immunohistochemistry; (3) studies assessing the correlation of TLSs with survival or other clinicopathologic indicators and gene expression in BCa; (4) full text, original research article published in English (alternatively, for articles published in Chinese, the English title and abstract were available); and (5) favorable studies selected from similar works published by the same term. The exclusion criteria were as follows: (1) conference abstracts, letters to the editor, reviews, comments, and animal trials; (2) studies with sample sizes $<50$, since a small sample size induces publication bias; and (3) work without raw data that could not be traced.

\section{Data extraction and quality assessment}

The incorporated studies' baseline information, including the name of the first author, country of study, publication year, sample size, TLS detection methods, TLS location, grouping methods, follow-up period, and outcome measures, was extracted (Table 1). Additionally, we recorded the original data of the clinicopathologic parameters and related gene expression data for analysis of the 
Table 1 Main information of the included studies

\begin{tabular}{|c|c|c|c|c|c|c|c|c|}
\hline Author (ref.) & Publishing year & country & No. of patients & $\begin{array}{l}\text { TLSs detection } \\
\text { methods }\end{array}$ & TLSs location & $\begin{array}{l}\text { Grouping } \\
\text { methods }\end{array}$ & $\begin{array}{l}\text { Follow-up } \\
\text { (months) } \\
\text { Median (range) }\end{array}$ & Outcome \\
\hline $\begin{array}{l}\text { Figenschau et al. } \\
{[15]}\end{array}$ & 2015 & Norway & 290 & $\begin{array}{l}\text { IHC for T, B cells } \\
\text { and HEV }\end{array}$ & Global & $\begin{array}{l}\text { Negative/posi- } \\
\text { tive }\end{array}$ & NA & NA \\
\hline Kim et al. [17] & 2016 & Korea & 204 & $\begin{array}{l}\text { IHC for T, B cells } \\
\text { and HEV }\end{array}$ & $\begin{array}{l}\text { Tumor adjacent } \\
\text { tissues }\end{array}$ & $\begin{array}{l}\text { None, little, } \\
\text { moderate, or } \\
\text { abundant }\end{array}$ & NA & NA \\
\hline Zhou et al. [18] & 2016 & China & 100 & $\begin{array}{l}\text { IHC for T, B, DC } \\
\text { cells, and HEV }\end{array}$ & Global & $\begin{array}{l}\text { Negative/posi- } \\
\text { tive }\end{array}$ & NA & NA \\
\hline Lee et al. [19] & 2016 & Korea & 769 & $\begin{array}{l}\text { IHC for T and B } \\
\text { cells }\end{array}$ & $\begin{array}{l}\text { Tumor adjacent } \\
\text { tissues }\end{array}$ & $\begin{array}{l}\text { None, little, } \\
\text { moderate, or } \\
\text { abundant }\end{array}$ & $\begin{array}{l}47 \text { years }(23-76 \\
\text { years) }\end{array}$ & OS, DFS \\
\hline $\begin{array}{l}\text { Laurence et al. } \\
\text { [20] }\end{array}$ & 2017 & Belgium & 125 & $\begin{array}{l}\text { IHC for T and B } \\
\text { cells }\end{array}$ & Global & $\begin{array}{l}\text { Negative/posi- } \\
\text { tive }\end{array}$ & NA & NA \\
\hline Gao et al. [21] & 2017 & China & 150 & $\begin{array}{l}\text { IHC for T, B, DC } \\
\text { cells, and HEV }\end{array}$ & Global & $\begin{array}{l}\text { Negative/posi- } \\
\text { tive }\end{array}$ & NA & NA \\
\hline Liu et al. [22] & 2017 & China & 248 & $\begin{array}{l}\text { IHC for T and B } \\
\text { cells }\end{array}$ & Global & $\begin{array}{l}\text { Negative/posi- } \\
\text { tive }\end{array}$ & $78(1-134)$ & DFS \\
\hline Lee et al. [23] & 2019 & Korea & 335 & $\begin{array}{l}\text { IHC for T, B cells } \\
\text { and HEV }\end{array}$ & $\begin{array}{l}\text { Tumor adjacent } \\
\text { tissues }\end{array}$ & $\begin{array}{l}\text { None, little, } \\
\text { moderate, or } \\
\text { abundant }\end{array}$ & NA & OS \\
\hline Chao et al. [24] & 2020 & China & 60 & ${ }^{H} \mathrm{HC}$ for T, B cells & $\begin{array}{l}\text { Tumor adjacent } \\
\text { tissues }\end{array}$ & $\begin{array}{l}\text { None, little, } \\
\text { moderate, or } \\
\text { abundant }\end{array}$ & $48(22-163)$ & DFS \\
\hline
\end{tabular}

$T L S$ s tertiary lymphoid structures, HEV high endothelial venule, $N A$ not available

relationship with TLSs in a predefined table. The hazard ratios (HRs) and their associated 95\% confidence intervals (95\% CIs) from multivariate analysis were extracted. The Newcastle-Ottawa Scale (NOS) was used to evaluate the study quality. Two authors (NNZ and FJQ) independently conducted the process and resolved disagreements through discussion with more researchers. The NOS, ranging from 0 to 9 , includes three domains: selection of the exposed cohort, comparability of the cohorts, and assessment of the outcome. Studies with a NOS score greater than 6 were considered high-quality studies.

\section{Evaluation and collection of clinicopathological parameters and gene expression data for $\mathrm{BCa}$}

We traced and collected common clinicopathological data for $\mathrm{BCa}$, including patient age, tumor size, tumor differentiation status, pTNM stage, lymph node metastasis status, lymphovascular invasion status, and TIL density. We collected the original data and divided each cohort into a high and low group for each indicator to ensure the uniformity, rationality, and accuracy of the $\mathrm{P}$ values from further Chi-square tests. Similar to the classification standards adopted in most incorporated articles, we classified the $\mathrm{BCa}$ patients into a young and an old group according to the cutoff age of 50, a large and a small group (small tumor size $\leq 20 \mathrm{~mm}$, large tumor size $>20 \mathrm{~mm})$, a well-differentiated group $(\mathrm{G} 1+\mathrm{G} 2)$ and a poorly differentiated group $(\mathrm{G} 3+\mathrm{G} 4)$, an early-stage (I+II) group and a late-stage (III + IV) group, and positive and negative lymph node metastasis and lymphovascular invasion groups. Additionally, we grouped patients by TIL density into a low and a high group according to the mean or the median value, whichever was provided, and the patients were also grouped according to the absence or presence of TLSs. Patients were classified by the expression of related genes, including HER2 (human epidermal growth factor receptor 2), ER (estrogen receptor), PR (progesterone receptor), and Ki67, into positive and negative groups. Fisher's method, which combines $p$ values, is appropriate for analysis of this type of data. The original data and $p$ values from chi-square tests in the included studies are shown in detail in Additional file 1: Tables S1 and S2, respectively.

\section{Statistical analysis}

Statistical software version 16.0 (Stata Corporation, College Station, TX, USA) was used to perform the analysis, while HR and 95\% CI data were log transformed and pooled. The fixed effects model was selected if $I^{2}<50 \%$, and the random effects model was considered if significant heterogeneity existed $\left(I^{2}>50 \%\right)$. The heterogeneity among the studies was evaluated by the $I^{2}$ statistic proposed by Higgins and the Cochran Q test. The risk of death of BCa patients with a higher density of TLSs was 
evaluated by $\mathrm{HRs}$ and $95 \%$ CIs. A $\mathrm{HR}<1$ indicated a better prognosis. Publication bias was evaluated by visually assessing the asymmetry of an inverted funnel plot and was quantified by Egger's and Begg's tests. We combined the $p$ values from the chi-square test of each included study by Fisher's method combined with $p$ value analysis. $p<0.05$ indicated that TLSs were statistically related to a certain clinicopathologic parameter or the expression of a certain gene.

\section{Results}

\section{Study selection and characteristics}

Using the described search strategy, we identified 574 records in the primary literature search. After screening the titles, abstracts, and methods of each publication, 528 studies were excluded, as they were reviews, animal studies, or irrelevant studies. We carefully assessed the eligibility of each article based on the full text. Finally, 9 studies [15, 17-24] investigating the correlation between
TLSs and BCa patient outcomes and including other relevant clinicopathologic parameters or gene expression were included in this meta-analysis comprising 2281 cases. Figure 1 shows the flow diagram for literature retrieval and selection.

Table 1 summarizes the basic information and main characteristics of the 9 included studies. Among the 9 included articles, 4 studies evaluated the prognostic value of TLSs in BCa: 3 studies evaluated disease-free survival (DFS) $[19,22,24]$, and 2 studies evaluated overall survival (OS) $[19,23]$. Six studies assessed the relationship of TLSs with other clinicopathologic parameters $[15,17$, 18, 20-22]. Four studies included patient age and tumor size $[15,18] ; 6$ studies included tumor grade $[15,17,18$, 20-22]; 2 studies included pTNM stage [18, 21]; 5 studies included lymph node metastasis status [15, 18, 20-22]; one study included lymphovascular invasion status [22]; and 2 studies included TIL density [20, 22]. Four studies evaluated the association between the expression

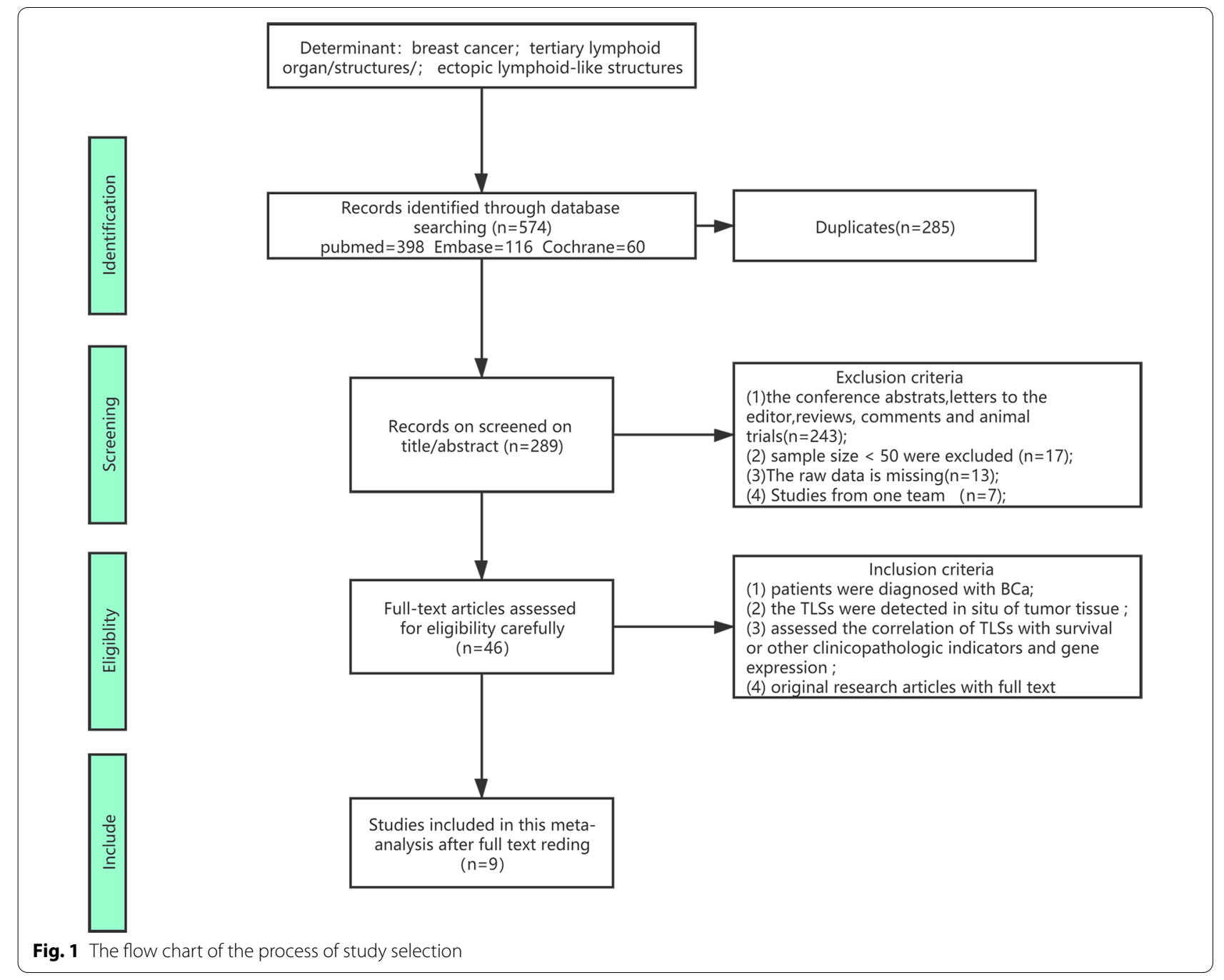


of related genes and TLSs [15, 17, 20, 22]. Four studies included Her2 expression [15, 17, 20, 22]; 3 studies included ER expression [15, 20, 22]; 3 studies included PR expression [15, 20, 22]; and 2 studies included Ki67 expression [20, 22]. The NOS scores of the included studies ranged from 5 to 9 and are shown in detail in Fig. 2 and Additional file 1: Table S3.

\section{Prognostic value of TLSs in BCa patients}

Four studies evaluated the prognostic value of TLSs in BCa [19, 22-24]. Three studies analyzed the prognostic value of TLSs for DFS in BCa [19, 22-24]. The random effects model was adopted: the heterogeneity among the 3 included studies was greater than $50 \%$ (Cochrane's Q, $\left.p=0.052, I^{2}=66.2 \%\right)$. The pooled results of the 3 studies comprising 1077 patients revealed that TLSs were significantly correlated with better DFS (HR $=0.40,95 \%$ CI: $0.17-0.93, p<0.001$; Fig. 3A). The sensitivity analysis confirmed the pooled results' stability and credibility, as removal of any cohort failed to change the statistical significance (Fig. 3B). Egger's and Begg's tests showed no significant publication bias $(p=0.121, p=1.000$; Fig. $3 C$,

A

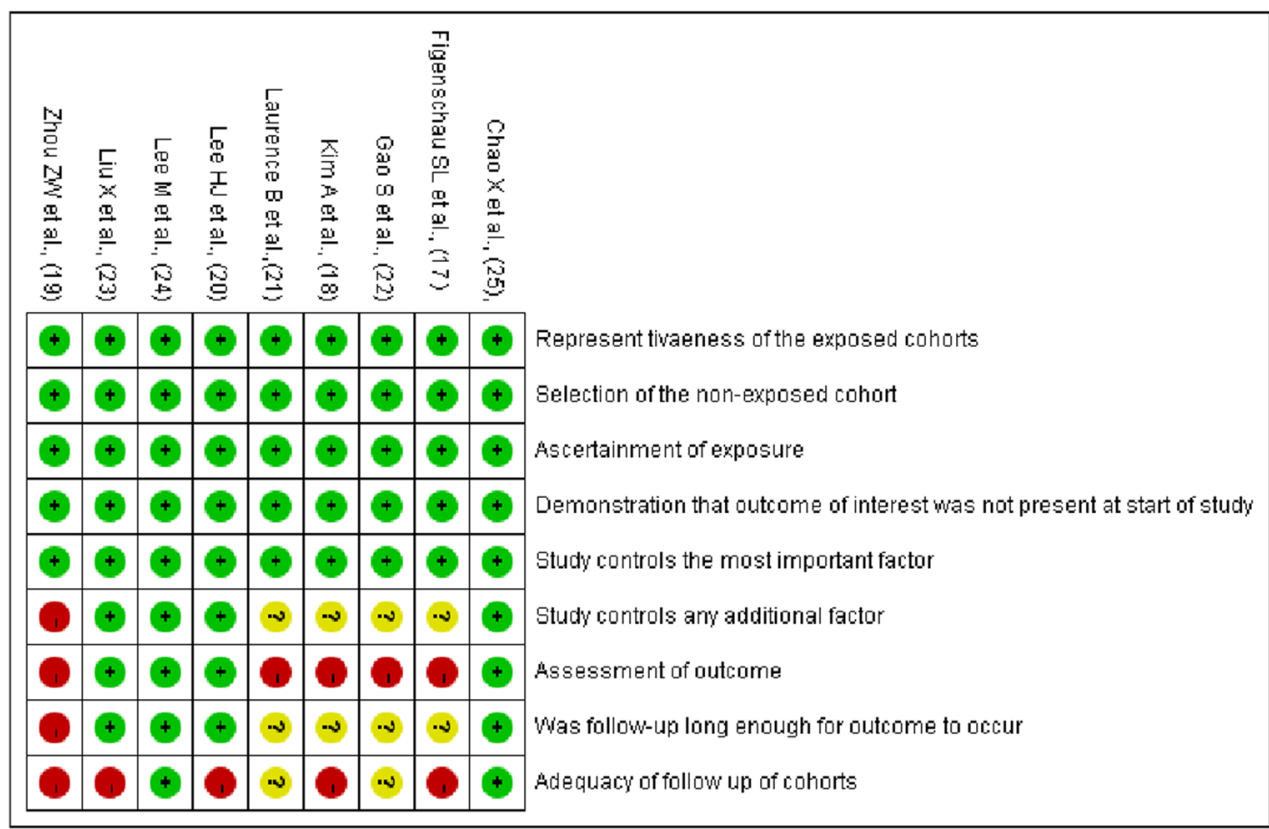

B

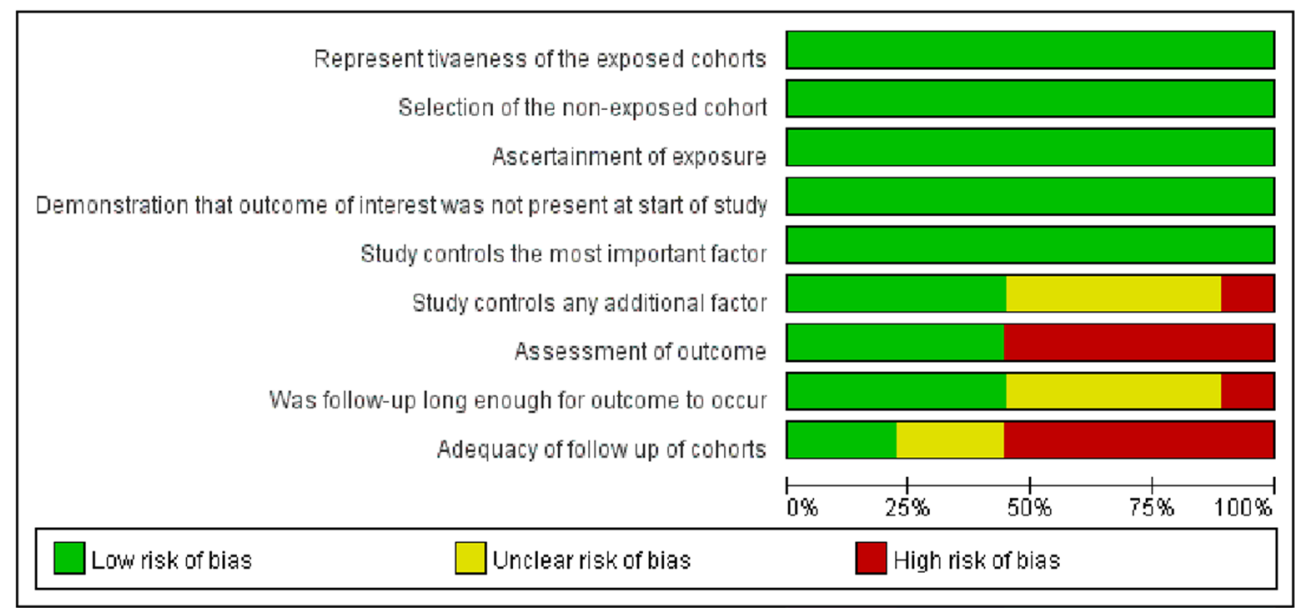

Fig. 2 A the risk of bias summary plot shows the risk for each of the included studies. The green, yellow, and red point represent low, unclear, and high risk, respectively. $\mathbf{B}$ the risk of graph bias showed the risk of bias item presented as percentages across all included studies, including increased risk, low risk, and unclear risk 
A

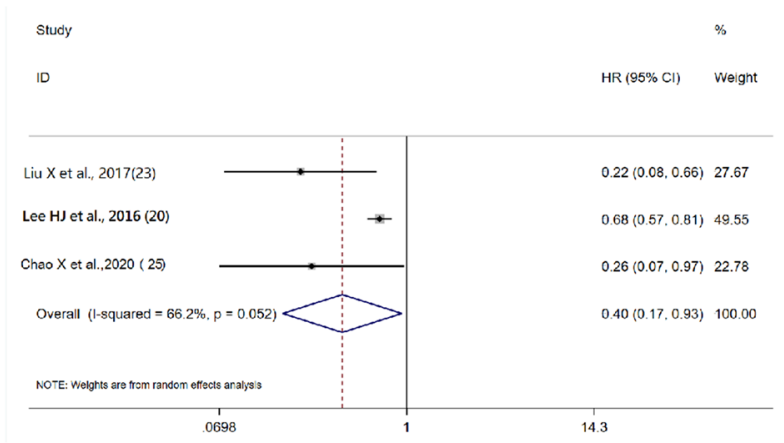

C Egger's publication bias plot

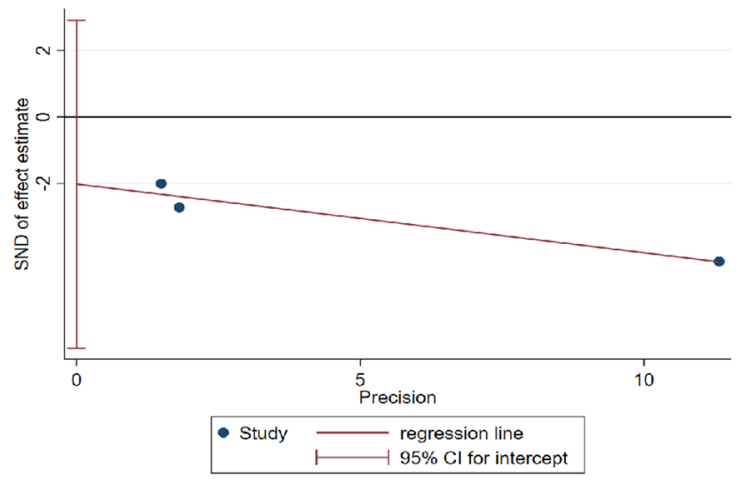

E

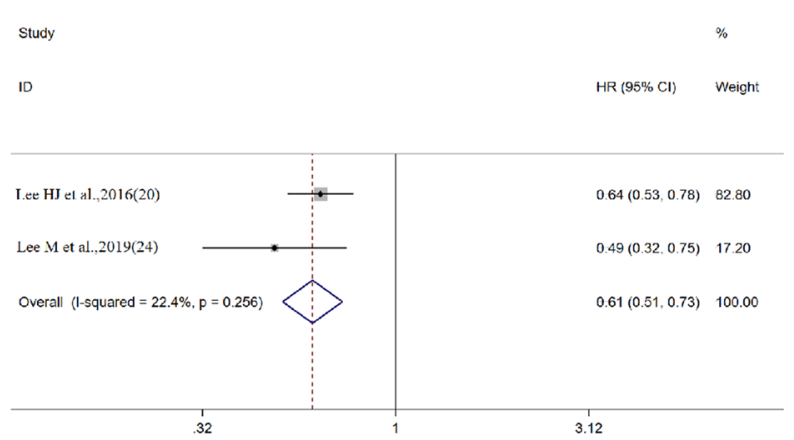

B

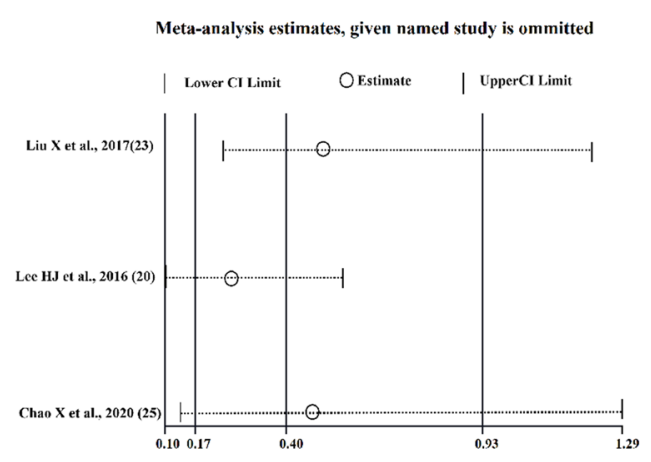

D

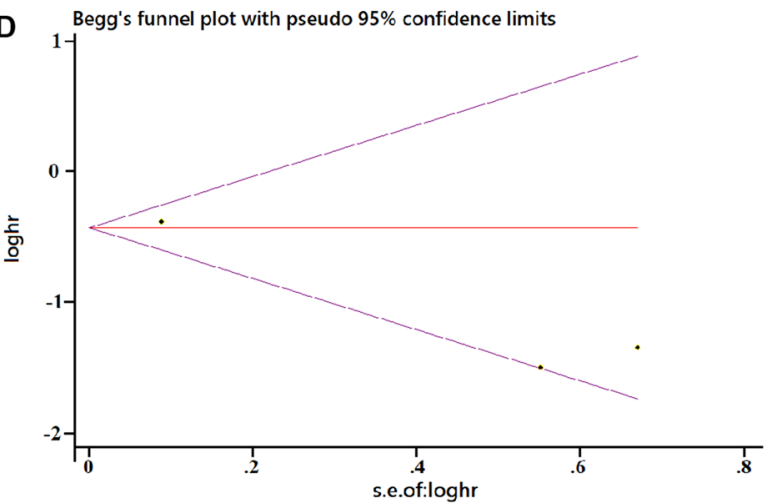

$\mathbf{F}$

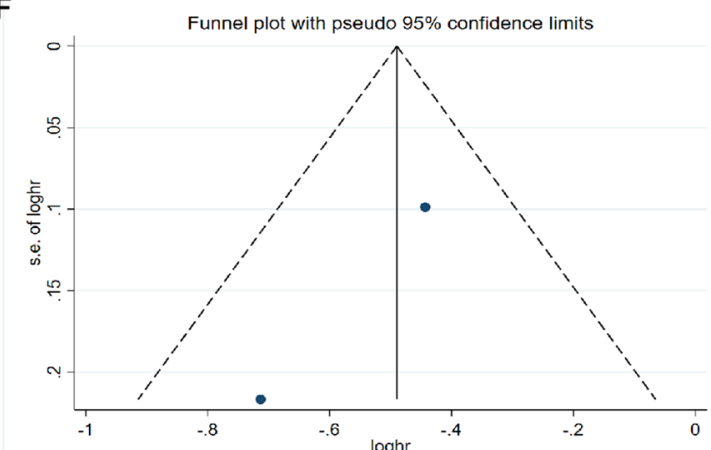

Fig. 3 A-D The forest plot,sensitivity analysis plot, Egger's test and Begg's test of the meta-analysis of DFS for BCa patients. E, FThe forest plot and funnel plot of the meta-analysis of OS for the BCa patients, from Cox multivariate analysis

D), indicating that a higher density of TLSs significantly predicted better DFS for BCa patients.

Only 2 studies $[19,23]$ evaluated the prognostic value of TLSs for OS in $\mathrm{BCa}$. The fixed effects model was appropriate owing to the lower heterogeneity among the included studies (Cochrane's $\mathrm{Q}, p=0.266 ; I^{2}=22.4 \%$ ). The results showed that a higher density of TLSs was a favorable prognostic factor for OS in $\mathrm{BCa}$ patients $(\mathrm{HR}=0.61,95 \% \mathrm{CI}: 0.51-0.73, p<0.001$; Fig. $3 \mathrm{E})$, and the funnel plot in Fig. 3F indicated no publication bias. Information on the analysis of DFS and OS in patients with $\mathrm{BCa}$ is shown in Table 2.

\section{The association of TLSs with clinicopathological} parameters of $\mathrm{BCa}$

Table 3 shows the final results of the correlation analysis between TLSs and various clinicopathological parameters. Four studies $[15,18,20,21]$ analyzed the relationship between TLSs and patient age, and the results indicated no significant correlation between TLSs and patient age $\left(x^{2}=5.332, p=0.722\right)$. Similarly, no significant correlation was found in the 4 studies $[15,18,20$, 21] that analyzed the relationship between TLSs and tumor size $\left(\chi^{2}=4.149, p=0.843\right)$. Tumor differentiation status $\left(X^{2}=70.765, p<0.001\right)$, pTNM stage $\left(\chi^{2}=19.690\right.$, 
Table 2 Pooled HR, heterogeneity, and publication bias of the meta-analysis of DFS and OS in BCa

\begin{tabular}{|c|c|c|c|c|c|c|c|c|c|}
\hline \multirow[t]{2}{*}{ Factor } & \multirow{2}{*}{$\begin{array}{l}\text { Include } \\
\text { studies }\end{array}$} & \multirow[t]{2}{*}{ Cases } & \multirow[t]{2}{*}{ Model } & \multirow[t]{2}{*}{ Pooled HR (95\% Cl) } & \multirow[t]{2}{*}{$p$} & \multicolumn{2}{|c|}{ Publication bias } & \multicolumn{2}{|c|}{ Heterogeneity } \\
\hline & & & & & & Egger & Begg & $I^{2}$ & $p$ \\
\hline DFS & 3 & 1077 & R & $0.40(0.17-0.93)$ & $<0.001$ & 0.121 & 1.000 & $66.2 \%$ & 0.052 \\
\hline OS & 2 & 1104 & $\mathrm{~F}$ & $0.61(0.42-0.90)$ & $<0.001$ & - & - & $22.4 \%$ & 0.256 \\
\hline
\end{tabular}

Table 3 The fisher's method combine p-value analysis of the correlation between TLS and various clinicopathological parameters and gene expression

\begin{tabular}{|c|c|c|c|c|c|}
\hline Parameters & No. of cohorts & Number of cases & $x^{2}$ value & $p$ & Relationship with TLSs \\
\hline Patients'age & 4 & 665 & 5.332 & 0.722 & No statistical significance \\
\hline Tumor size & 4 & 665 & 4.149 & 0.843 & No statistical significance \\
\hline Tumor differentiation & 6 & 1117 & 70.765 & $<0.001$ & Negative \\
\hline pTNM stage, & 2 & 250 & 19.690 & $<0.001$ & Negative \\
\hline Lymph node metastasis & 5 & 913 & 31.939 & $<0.001$ & Negative \\
\hline TILs density & 2 & 373 & 27.631 & $<0.001$ & Positive \\
\hline HER2 & 4 & 867 & 42.448 & $<0.001$ & Positive \\
\hline ER & 3 & 663 & 33.098 & $<0.001$ & Negative \\
\hline$P R$ & 3 & 663 & 35.455 & $<0.001$ & Negative \\
\hline Ki67 & 2 & 373 & 13.468 & 0.009 & Positive \\
\hline
\end{tabular}

$p<0.001)$, lymph node metastasis status $\left(\mathrm{x}^{2}=31.939\right.$, $p<0.001)$, and TIL density $\left(x^{2}=27.631, p<0.001\right)$ were found to be significantly correlated with TLSs. One paper [22] reported the relationship between TLSs and lymphovascular invasion without finding statistical significance $\left(x^{2}=3.647, p<0.056\right.$, Additional file 1: Table S1).

\section{The association of TLSs and related gene expression of $\mathrm{BCa}$}

We also investigated the expression of key genes (HER2, ER, and PR) affecting the diagnosis, therapy selection, and prognosis of $\mathrm{BCa}$ to evaluate the relationship of these genes with TLSs. A total of 4 papers [15, 17, 20, 22], 3 papers $[15,20,22]$ and 3 papers $[15,20,22]$ reported the association of HER-2, ER, and PR, respectively, with TLSs. Positive correlations were found between these genes (HER-2, $\chi^{2}=42.448, p<0.001 ; \mathrm{ER}, \chi^{2}=33.098$, $p<0.001$; PR, $\left.\chi^{2}=35.455, p<0.001\right)$ and TLSs. Two articles [20, 22] documented the relationship between Ki67 (reflecting the tumor proliferation index) and TLSs, and the analysis results showed that TLSs were also significantly correlated with Ki67 expression $\left(\chi^{2}=13.468\right.$, $p=0.009$, Table 3).

\section{Discussion}

Recent studies have highlighted the role of TLSs in the immunotherapeutic response of malignant tumors [12, 13]. In general, the presence of TLSs is an indicator of favorable prognosis in many solid tumors. However, some works have reported a negative prognostic impact of TLSs. Previous studies reported that the presence of TLSs was significantly associated with a more advanced stage in colorectal cancer [25] and a higher tumor grade, as well as a higher frequency of lymph node metastasis, in $\mathrm{BCa}$ [15]. These inconsistent results may be due to the nonuniform evaluation methods adopted in these studies. Therefore, we assessed the prognostic role and clinicopathological impact of TLSs in this meta-analysis incorporating 9 different cohorts containing a total of 2281 patients with BCa. Although only 4 studies evaluated the prognostic value of TLSs, the presence of TLSs was found to be an independent favorable prognostic factor for OS and DFS in BCa, indicating that the TLS status in tumor tissue is essential for predicting the clinical outcomes of BCa. Previous literature has recorded that "TLSs could provide a favorable milieu for the education of intratumoral CD8 $+\mathrm{T}$ cells and B cells against cancer"[11]. Generally, TLSs represent sites of lymphoid neogenesis characterized by mature dendritic cells in a $\mathrm{T}$ cell zone adjacent to a $\mathrm{B}$ cell follicle including a germinal center. Recently, investigators developed a semantic segmentation model for whole-slide histopathological images, named HookNet, which can segment TLSs and germinal centers in lung cancer [26]. Inflammatory BCa (IBC) is an aggressive form of this disease, and it shows higher expression of TLS signatures with higher sensitivity to immune checkpoint inhibitors [27]. Therefore, the formation of TLSs indicates a vigorous rather than an exhausted immune response. Attempts to induce the 
formation or modulate the function of TLSs may offer new therapeutic opportunities for cancer patients.

Recent advances in tumor immunology have shown that the tumor microenvironment is in a state of dynamic evolution [28]. The complex interactions among immune and tumor cells are crucial for tumor progression [29]. A recent study reported that lymphocyte clusters (LCs), like TLSs, are closer to tumor cell islands in patients with a good outcome, with a decrease in the LC size with decreasing distance to tumor cell islands [30]. Thus, it is crucial to identify the clinicopathological impact of TLSs in BCa. This meta-analysis first analyzed the relationships between TLSs and other clinicopathological indicators. Our results showed that TLSs were not significantly correlated with patient age or tumor size but markedly influenced the clinicopathological indicators that reflected tumor progression (e.g., tumor differentiation status, pTNM stage, lymph node metastasis status, and lymphovascular invasion status), suggesting that TLSs may be closely related to tumor progression and evolution. In addition, we found that the density of TILs displayed a positive correlation with the absence of TLSs. A study in colorectal cancer reported that TLSs were linearly associated with lymphocyte infiltration. TLSs provide a pathway for recruitment of TILs and cooperate with TILs in a coordinated antitumor immune system response, predicting low-risk, early-stage colorectal cancer [31].

It is well known that the development of $\mathrm{BCa}$ is strongly associated with multiple gene aberrations. Molecular analysis can enhance the understanding of the biological behavior of BCa. The key molecules, HER-2, ER, and $\mathrm{PR}$, have been found to play a critical role in the diagnosis, therapy selection, monitoring, and prognosis of $\mathrm{BCa}$. Thus, the correlations between these critical molecules and TLSs were analyzed in the present meta-analysis, and significant correlations were identified. It was found that the monoclonal antibody trastuzumab was associated with a complete or partial response in patients with HER-2-positive tumors, characterized by higher infiltration of leukocytes and an augmented capacity to mediate antibody-dependent cellular cytotoxicity, compared with patients with nonresponding tumors [32]. TLSs have been reported to be associated with significantly better DFS in HER-2 + IBC but not in HER-2- IBC [22]. Since tumoral TLSs can promote more effective antitumor immunity [33, 34], and an active immune response is important for treatment of HER-2 + IBC, it was documented that many HER $-2+\mathrm{BCa}$ patients are treated with chemotherapy and/or HER-2 targeted therapy and that some of the favorable effects are attributed to active antitumor immunity [35-37]. Passive immunotherapy, particularly with trastuzumab and pertuzumab, is an effective therapeutic strategy in HER2 + BCa [38].
Therefore, the presence of TLSs might be an indicator of the treatment response in Her2 $+\mathrm{BCa}$ patients. And one study explored the association of TLSs and drug responses in $\mathrm{BCa}$ patients, but not directly explored their relationship. The study found a higher frequency of TLSs in IBC than non-IBC, and the IBC were more sensitive to ICIs than non-IBC [27]. It indicates that a higher frequency of TLSs in BCa tissue might be vulnerable to checkpoint inhibitors (ICIs). Another study found TLSs in the $\mathrm{BCa}$ were infiltrated with PD-L1+, PD-L2+, LAG3 +, and TIM3 + (immune checkpoint molecule) cells, which has an important implication for the successful response to the immunotherapy [39]. These observations suggest that TLSs are important site of immune activation and regulation, particularly in tumors with extensive baseline immune infiltration. While whether TLSs can predict the effect of immunotherapy is worthy of further exploration and validation by the large sample clinical trials.

ER and PR are used as indicators to evaluate prognosis and guide clinical endocrine therapy in $\mathrm{BCa}$. An in silico analysis of gene expression profiles in 2976 nonmetastatic BCa samples showed that ER- and PR-positive BCa tissues were infiltrated with their characteristic immune components and directly affected the prognosis of $\mathrm{BCa}$ patients [40]. Cell proliferation is a basis for tumorigenesis, and Ki67, a nuclear antigen associated with proliferating cells, is an important marker for effectively evaluating the proliferative activity of tumor cells [41]. TLSs were also significantly correlated with Ki67 expression, although only two documents reported this connection. In addition, the advent of new genetic tests has emphasized the role of Ki67 as a prognostic and predictive marker in $\mathrm{BCa}$. Specialists have reassessed evidence that could change guidelines to include Ki67 in the standard pathological assessment of BCa [42]. The summary of these analyses revealed significant correlations between TLSs and various indicators, reflecting tumor biological behavior and affecting tumor diagnosis, therapy selection, monitoring, and prognosis. Notably, accurate detection of the number of TLSs is based on large pathological tissues, since extensive sampling can allow detection of more morphological structures. Studies have proven that intratumoral TLSs are an indicator of favorable prognosis in patients with pancreatic cancer [43], while peritumoral TLSs correlate with protective immunity and improved prognosis in patients with hepatocellular carcinoma [44], indicating the significance of TLSs localization in cancer patients. However, information on the specific histomorphological location is more dependent on extensive sampling; thus, larger pathological tissues imply a higher accuracy of morphological information and are of great importance for predicting the prognosis of patients. 


\section{Conclusions}

In summary, TLSs have attracted great interest among researchers in the field of tumor immunology. Our analysis results indicated that a high level of TLSs was significantly correlated with favorable outcomes for $\mathrm{BCa}$ patients. In addition, there were significant correlations among the clinicopathological indicators, expression of critical genes and presence of TLSs. Thus, TLSs are expected to be a biomarker for predicting prognosis and guiding immunotherapy in $\mathrm{BCa}$ and have great significance for the development of individualized treatment.

\begin{abstract}
Abbreviations
TLSs: Tertiary lymphoid structures; BCa: Breast cancer; IBC: Inflammatory breast cancer; OS: Overall survival; DFS: Disease-free survival; ICB: Immune checkpoint blockade; ICls: Checkpoint inhibitors; LCs: Lymphocyte clusters; TILs: Tumor infiltrating lymphocytes; TMEs: Tumor microenvironments; HER2: Human epidermal growth factor receptor 2; ER: Estrogen receptor; PR: Progesterone receptor.
\end{abstract}

\section{Supplementary Information}

The online version contains supplementary material available at https://doi. org/10.1186/s12935-021-02242-x.

Additional file 1: Table S1. The original data and chi-square test for the TLSs and clinicopathologic parameters. Table S2 The original data and chi-square test for the TLSs and related gene expression. Table S3. NOS scores of included studies.

\section{Acknowledgements}

All authors have contributed significantly. All authors are in agreement with the content of the manuscript.

\section{Authors' contributions}

NNZ and FJQ designed, wrote and reviewed the manuscript. HL and ZJL performed the literature search, literature selection, and data extraction. YCZ and XH checked the data extraction. NNZ and FJQ statistically analyzed the obtained data. All authors contributed to the preparation of the manuscript. All authors read and approved the final manuscript.

\section{Funding}

This work was supported by the National key research and development projects of China (No. 2018YFC0115300); National Natural Science Foundation of China (No. 81727802).

\section{Availability of data and materials}

Not applicable.

\section{Declarations}

Ethics approval and consent to participate

Not applicable.

\section{Consent for publication}

All authors are in agreement with the publication of the manuscript.

\section{Competing interests}

The authors declare that they have no competing interests.

\section{Author details}

${ }^{1}$ Center for Regenerative and Reconstructive Medicine, Med-X Institute of Western China Science and Technology Innovation Harbour, The First
Affiliated Hospital of Xi'an Jiaotong University, Xi'an 710049, Shaanxi, China. ${ }^{2}$ National Local Joint Engineering Research Center for Precision Surgery and Regenerative Medicine, The First Affiliated Hospital of Xi'an Jiaotong University, Xi'an 710061, Shaanxi, China. ${ }^{3}$ Department of Hepatobiliary Surgery, First Affiliated Hospital, Xi'an Jiaotong University, Xi'an 710061, Shaanxi, China.

Received: 2 July 2021 Accepted: 1 October 2021

Published online: 15 October 2021

\section{References}

1. DeSantis CE, Ma J, Gaudet MM, et al. Breast cancer statistics, 2019. CA A Cancer J Clin. 2019;69(6):438-51.

2. Greaney ML, Sprunck-Harrild K, Ruddy KJ, et al. Study protocol for Young \& Strong: a cluster randomized design to increase attention to unique issues faced by young women with newly diagnosed breast cancer. BMC Public Health. 2015;15:37.

3. Siegel RL, Miller KD, Jemal A. Cancer statistics, 2020. CA A Cancer J Clin. 2020;70(1):7-30.

4. Tang H, Qiao J, Fu YX. Immunotherapy and tumor microenvironment. Cancer Lett. 2016:370(1):85-90.

5. Heinhuis KM, Ros W, Kok M, et al. Enhancing antitumor response by combining immune checkpoint inhibitors with chemotherapy in solid tumors. Ann Oncol. 2019;30(2):219-35.

6. Adams S, Schmid P, Rugo HS, et al. Pembrolizumab monotherapy for previously treated metastatic triple-negative breast cancer: cohort A of the phase II KEYNOTE-086 study. Ann Oncol. 2019;30(3):397-404.

7. Dirix LY, Takacs I, Jerusalem G, et al. Avelumab, an anti-PD-L1 antibody, in patients with locally advanced or metastatic breast cancer: a phase $1 \mathrm{~b}$ JAVELIN Solid Tumor study. Breast Cancer Res Treat. 2018;167(3):671-86.

8. Nanda R, Chow LQ, Dees EC, et al. Pembrolizumab in patients with advanced triple-negative breast cancer: phase Ib KEYNOTE-012 study. J Clin Oncol. 2016;34(21):2460-7.

9. Adams S, Loi S, Toppmeyer D, et al. Pembrolizumab monotherapy for previously untreated, PD-L1-positive, metastatic triple-negative breast cancer: cohort B of the phase II KEYNOTE-086 study. Ann Oncol. 2019;30(3):405-11.

10. Sautes-Fridman C, Petitprez F, Calderaro J, et al. Tertiary lymphoid structures in the era of cancer immunotherapy. Nat Rev Cancer. 2019;19(6):307-25.

11. Colbeck EJ, Ager A, Gallimore A, et al. Tertiary lymphoid structures in cancer: drivers of antitumor immunity, immunosuppression, or bystander sentinels in disease? Front Immunol. 2017;8:1830.

12. Helmink BA, Reddy SM, Gao J, et al. B cells and tertiary lymphoid structures promote immunotherapy response. Nature. 2020;577(7791):549-55.

13. Cabrita R, Lauss M, Sanna A, et al. Tertiary lymphoid structures improve immunotherapy and survival in melanoma. Nature. 2020;577(7791):561-5.

14. Dieu-Nosjean MC, Giraldo NA, Kaplon H, Germain C, Fridman WH, Sautes-Fridman C. Tertiary lymphoid structures, drivers of the anti-tumor responses in human cancers. Immunol Rev. 2016;271(1):260-75.

15. Figenschau SL, Fismen S, Fenton KA, et al. Tertiary lymphoid structures are associated with higher tumor grade in primary operable breast cancer patients. BMC Cancer. 2015;15:101.

16. Sofopoulos M, Fortis SP, Vaxevanis CK, et al. The prognostic significance of peritumoral tertiary lymphoid structures in breast cancer. Cancer Immunol Immunother. 2019;68(11):1733-45.

17. Kim A, Heo SH, Kim YA, et al. An examination of the local cellular immune response to examples of both ductal carcinoma in situ (dcis) of the breast and dcis with microinvasion, with emphasis on tertiary lymphoid structures and tumor infiltrating lymphoctytes. Am J Clin Pathol. 2016;146(1):137-44.

18. Zou Zhongwei. Expression of tertiary lymphoid structures in breast cancer and its clinical significance [Master dissertation]: University of South China. 2016.

19. Lee HJ, Park IA, Song IH, et al. Tertiary lymphoid structures: prognostic significance and relationship with tumour-infiltrating lymphocytes in triple-negative breast cancer. J Clin Pathol. 2016;69(5):422-30. 
20. Buisseret L, Garaud S, de Wind A, et al. Tumor-infiltrating lymphocyte composition, organization and PD-1/ PD-L1 expression are linked in breast cancer. Oncoimmunology. 2017;6(1):e1257452.

21. Gao Song. Expression and clinical significance of CD4 +, CD8 + lymphocytes and tertiary lymphoid structures in breast cancer tissues [Master dissertation]: University of South China. 2017.

22. Liu X, Tsang J, Hlaing T, et al. Distinct tertiary lymphoid structure associations and their prognostic relevance in HER2 positive and negative breast cancers. Oncologist. 2017:22(11):1316-24.

23. Lee $\mathrm{M}$, Heo $\mathrm{SH}$, Song $\mathrm{IH}$, et al. Presence of tertiary lymphoid structures determines the level of tumor-infiltrating lymphocytes in primary breast cancer and metastasis. Mod Pathol. 2019;32(1):70-80.

24. Chao X, Liu L, Sun P, et al. Immune parameters associated with survival in metaplastic breast cancer. Breast Cancer Res. 2020;22(1):92.

25. Bento DC, Jones $\mathrm{E}$, Junaid $\mathrm{S}$, et al. High endothelial venules are rare in colorectal cancers but accumulate in extra-tumoral areas with disease progression. Oncoimmunology. 2015;4(3):e974374

26. van Rijthoven M, Balkenhol M, Silina K, van der Laak J, Ciompi F. HookNet: Multi-resolution convolutional neural networks for semantic segmentation in histopathology whole-slide images. Med Image Anal. 2021;68:101890.

27. Bertucci F, Boudin L, Finetti P, et al. Immune landscape of inflammatory breast cancer suggests vulnerability to immune checkpoint inhibitors. Oncoimmunology. 2021;10(1):1929724.

28. Rosenthal R, Cadieux EL, Salgado R, et al. Neoantigen-directed immune escape in lung cancer evolution. Nature. 2019;567(7749):479-85.

29. Locy H, de Mey S, de Mey W, et al. Immunomodulation of the tumor microenvironment: turn foe into friend. Front Immunol. 2018;9:2909.

30. Wortman JC, He TF, Solomon S, et al. Spatial distribution of B cells and lymphocyte clusters as a predictor of triple-negative breast cancer outcome. NPJ Breast Cancer. 2021:7(1):84.

31. Di Caro G, Bergomas F, Grizzi F, Doni A, Bianchi P, Malesci A, et al. Occurrence of tertiary lymphoid tissue is associated with $T$-cell infiltration and predicts better prognosis in early-stage colorectal cancers. Clin Cancer Res. 2014:20(8):2147-58.

32. Gennari R, Menard S, Fagnoni F, et al. Pilot study of the mechanism of action of preoperative trastuzumab in patients with primary operable breast tumors overexpressing HER2. Clin Cancer Res. 2004;10(17):5650-5.

33. de Chaisemartin L, Goc J, Damotte D, et al. Characterization of chemokines and adhesion molecules associated with $T$ cell presence in tertiary lymphoid structures in human lung cancer. Cancer Res. 2011;71(20):6391-9.

34. Munoz-Erazo L, Rhodes JL, Marion VC, Kemp RA. Tertiary lymphoid structures in cancer-considerations for patient prognosis. Cell Mol Immunol. 2020;17(6):570-5

35. Denkert C, von Minckwitz G, Brase JC, et al. Tumor-infiltrating lymphocytes and response to neoadjuvant chemotherapy with or without carboplatin in human epidermal growth factor receptor 2-positive and triple-negative primary breast cancers. J Clin Oncol. 2015;33(9):983-91.

36. Luen SJ, Salgado R, Fox S, et al. Tumour-infiltrating lymphocytes in advanced HER2-positive breast cancer treated with pertuzumab or placebo in addition to trastuzumab and docetaxel: a retrospective analysis of the CLEOPATRA study. Lancet Oncol. 2017;18(1):52-62.

37. Kwon MJ. Emerging immune gene signatures as prognostic or predictive biomarkers in breast cancer. Arch Pharm Res. 2019;42(11):947-61.

38. Arab A, Yazdian-Robati R, Behravan J. HER2-positive breast cancer immunotherapy: a focus on vaccine development. Arch Immunol Ther Exp (Warsz). 2020;68(1):2.

39. Solinas C, Garaud S, De Silva P, et al. Immune checkpoint molecules on tumor-infiltrating lymphocytes and their association with tertiary lymphoid structures in human breast cancer. Front Immunol. 2017:8:1412.

40. Zhang SC, Hu ZQ, Long JH, et al. Clinical implications of tumor-infiltrating immune cells in breast cancer. J Cancer. 2019;10(24):6175-84.

41. Penault-Llorca F, Radosevic-Robin N. Ki67 assessment in breast cancer: an update. Pathology. 2017:49(2):166-71.

42. Yerushalmi R, Woods R, Ravdin PM, et al. Ki67 in breast cancer: prognostic and predictive potential. Lancet Oncol. 2010;11(2):174-83.

43. Hiraoka N, Ino Y, Yamazaki-Itoh R, Kanai Y, Kosuge T, Shimada K. Intratumoral tertiary lymphoid organ is a favourable prognosticator in patients with pancreatic cancer. Br J Cancer. 2015;112(11):1782-90.

44. $\mathrm{Li} \mathrm{H}$, Liu H, Fu H, et al. Peritumoral tertiary lymphoid structures correlate with protective immunity and improved prognosis in patients with hepatocellular carcinoma. Front Immunol. 2021;12:648812.

\section{Publisher's Note}

Springer Nature remains neutral with regard to jurisdictional claims in published maps and institutional affiliations.
Ready to submit your research? Choose BMC and benefit from:

- fast, convenient online submission

- thorough peer review by experienced researchers in your field

- rapid publication on acceptance

- support for research data, including large and complex data types

- gold Open Access which fosters wider collaboration and increased citations

- maximum visibility for your research: over $100 \mathrm{M}$ website views per year

At $\mathrm{BMC}$, research is always in progress.

Learn more biomedcentral.com/submissions 